\title{
Stigmasterol Biosynthesis Pathway is Associated With the Dehiscence Process in Panax Ginseng Seeds
}

\author{
Jung Eun Min \\ Seoul National University College of Pharmacy \\ Nguyen Phuoc Long \\ Seoul National University College of Pharmacy \\ Ji Yeon Hong \\ Seoul National University College of Pharmacy \\ Sun Jo Kim \\ Seoul National University College of Pharmacy \\ Nguyen Hoang Anh \\ Seoul National University College of Pharmacy \\ Seul Ji Lee \\ Seoul National University College of Pharmacy \\ Sung Won Kwon ( $\nabla$ swkwon@snu.ac.kr) \\ Seoul National University College of Pharmacy \\ Jeong Hill Park \\ Seoul National University College of Pharmacy
}

\section{Research article}

Keywords: Ginseng seeds, Dehiscence, Dormancy release, Warm stratification, Squalene

Posted Date: September 4th, 2020

DOI: https://doi.org/10.21203/rs.3.rs-59650/v1

License: (c) (i) This work is licensed under a Creative Commons Attribution 4.0 International License. Read Full License 


\section{Abstract}

Background: Ginseng, officially known as Panax ginseng C.A. Meyer, has been traditionally used as a medicinal herb, especially in Asia. Ginseng is propagated from seeds, and seed germination difficulty makes it challenging to grow the species naturally in farms. P. ginseng seeds typically exhibit morphophysiological dormancy. Therefore, they require both morphological and physiological dormancy releases before germination. Although some studies have proposed methods of increasing seed germination rates, the underlying mechanisms of the seed dormancy release process remain unclear. In the present study, we investigated metabolic alterations during dehiscence in $P$. ginseng with the aim of unraveling their potential roles in dormancy release. We compared ginseng seed metabolome before and after dehiscing, in addition to the compositions of ginsenosides and phytosterols in the two periods and the presence of associated enzymes.

Results: Sugar, amino acid, and squalene concentrations were altered significantly after dehiscing. Phytosterols associated with the stigmasterol biosynthesis pathway increased after seed dehiscence, while ginsenosides and brassinosteroids were not altered significantly. In addition, squalene epoxidase, cycloartenol synthase, and 24-methylenesterol Cmethyltransferase were activated after seed dehiscence.

Conclusions: After seed dehiscence, the stigmasterol biosynthesis pathway was activated. Overall, our findings suggest that morphological activities that facilitate ginseng seed growth are the primary phenomena occurring during the dehiscence process. The findings of the present study enhance our understanding of $P$. ginseng germination processes and could promote further research on its germination and cultivation in farms.

\section{Background}

Ginseng (Panax ginseng C.A. Meyer) is one of the most widely used medicinal herbs. In particular, ginseng root has been reported to have various therapeutic effects, such as anti-cancer, immune response enhancement, blood glucose level improvement, and insulin regulation, among other properties [1-8]. Ginseng is propagated from seeds, and ginseng seeds exhibit morphophysiological dormancy according to Baskin's classification theory [9]. Freshly harvested seeds have very thick and hard seed coats in addition to underdeveloped embryo with physiological dormancy. Consequently, the two-step dormancy release process, warm and cold stratification, is essential for seed germination. Generally, right after seed harvesting, the seeds are stored in sand layers at $15-20^{\circ} \mathrm{C}$ for 3 months to allow warm stratification followed by storing at $4^{\circ} \mathrm{C}$ for 3 months for cold stratification [10]. In the course of warm stratification, the embryo begins to grow, and the seed coat dehisces [11]. Therefore, the achievement of warm stratification could be confirmed by seed coat dehiscence. Although the morphological alterations that occur under warm stratification are known, their underlying molecular mechanisms or associated pathways remain unclear. Investigating the underlying molecular activities during dehiscence could facilitate $P$. ginseng farming activities through an enhanced understanding of participating in germination in the medically important species.

Metabolomics is one of the "omics" approaches that aim to characterize all intermediates and products of metabolism in organisms. It can provide snapshots of an organism at one time, which reflect metabolic status [12, 13]. Therefore, metabolomics is an appropriate method of investigating metabolic phenotypes. Untargeted metabolomics is applied in numerous fields, such as biomarker discovery, disease diagnosis and prognosis, and geographical discrimination of plants [14-19]. Among the most extensively applied metabolomics techniques, gas chromatograph-mass spectrometry (GC-MS) is essential plant metabolome research. GC-MS based metabolomics can analyze volatile and non-volatile metabolites, especially primary metabolism products, in a single analysis. In addition, the retention indices and mass spectra are highly repeatable, and the available mass spectra libraries could facilitate the putative identification of GC-MS spectra peaks with high confidence [20]. Most metabolomic and transcriptomic studies on ginseng have focused on the roots [21-23], with hardly any such investigations conducted on the seeds, which are essential for the propagation of the highly valued medicinal plant. 
The present study aimed to investigate metabolic alterations during $P$. ginseng seed dehiscence and warm stratification. We first identified and validated differentially expressed metabolites and hypothesized the potential pathways associated with the dehiscence process. We also investigate phytosterol content and differential expression of genes before and after dehiscence. The results of the present study could facilitate our understanding of the underlying mechanisms of dormancy release in $P$. ginseng, and in turn, facilitate research on strategies of improving $P$. ginseng seed germination and cultivation.

\section{Results}

\section{Differentially expressed metabolites before and after seed dehiscing}

Overlaid chromatograms revealed clear patterns of peaks among different samples. However, the intensities of some peaks were quite distinct between the two groups. Principal component analysis (PCA) was performed to visualize the metabolic profiles of samples and the differences between the two groups, before dehiscence and after dehiscence groups (Fig. 1a). All 20 samples and seven quality control (QC) samples were separated on the PCA plot. Well-clustered QC samples and their low deviation values indicated the reliability of the results of the experiment. In addition, according to the heatmap analysis results, there were significant differences in metabolite expression patterns between the two groups (Fig. 1b). Kmeans clustering results also demonstrated that three groups of samples were categorized well (Additional file 1: Fig. S2).

$T$-test and partial least squares discriminant analysis (PLS-DA) were performed on the same datasets, and according to the $t$-test results, 72 metabolites exhibited significant alteration after seed dehiscence, with 29 metabolites increased, and 43 metabolites decreased (false discovery rate $[F D R]<0.001$ ). The descriptive model based PLS-DA also classified the two seed groups well. Accuracy, $\mathrm{R}^{2}$, and $\mathrm{Q}^{2}$ were close to the optimal values, suggesting distinction between the two groups (Fig. 1c). Twenty-three metabolites had variable important in projection (VIP) scores greater than 1.2; 15 metabolites were determined as biomarkers of before and after seed dehiscing, and they were amino acids, sugars, and their derivatives (Table 1).

To validate the metabolites as biomarkers of seed dehiscence, receiver operating characteristic (ROC) curve analysis was performed on another set of experimental results analyzed using new samples and based on the same sample treatment and study design. ROC curve analysis based on the new dataset and the 15 predicted biomarkers confirmed that two seed groups were well-differentiated (Fig. 2a), and the area under the curve results indicated that they were suitable biomarkers for ginseng dehiscence (Fig. 2b). The heatmap derived from hierarchical clustering analysis also revealed that the two groups had distinct metabolite profiles (Fig. 2c).

\section{Pathway enrichment analysis suggests squalene is crucial in ginseng seed dehiscence}

The 15 metabolites identified as potential biomarkers were subjected to pathway enrichment analysis to predict the potential metabolic pathways associated with seed dehiscence. Metabolic pathways associated with only nine metabolites were identified (Table 2). The nine metabolites are potentially involved in various metabolic pathways. For example, I-valine, I-leucine, and l-isoleucine, were associated with seven different pathways, while squalene and myo-inositol were only associated with "biosynthesis of secondary metabolites" and "metabolic pathways." In particular, squalene is a precursor of ginsenosides, the primary pharmacological ingredient in ginseng, and a plant steroid essential for plant growth. Its dynamics could facilitate the understanding of the underlying molecular mechanisms of seed dehiscence.

\section{Comparison of plant steroid composition}

Plant steroids, including phytosterols and brassinosteroids, are synthesized from squalene. Therefore, a decrease in squalene after seed dehiscence may influence the composition of the seed oil. Therefore, the unsaponifiable fraction in seed oil before and after ginseng seed dehiscing was compared using GC-MS (Fig. 3), and 12 phytosterols were identified using a combination of retention order and mass fragmentation data or authentic standards (Table 3 ). 
Comparison of retention order and mass fragmentation patterns with the findings of a similar previous study that analyzed

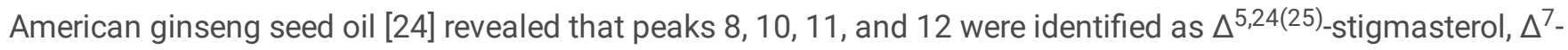
avenasterol, 24-methylenecycloartanol, and citrostadienol, respectively. Peaks 1-7 and 9 were confirmed as squalene, 2,3oxidosqualene, campesterol, stigmasterol, clerosterol, $\beta$-sitosterol, $\Delta^{5}$-avenasterol, and lupeol, respectively, by comparing retention times and mass spectra of their authentic standards. The eight phytosterols identified using standards were analyzed quantitatively, and 2,3-oxidosqualene was a major component of ginseng seed oil, followed by squalene, and $\Delta^{5}$ avenasterol. Based on the comparison between the pre and post-dehiscence groups, 2,3-oxidosqualene, $\Delta^{5}$-avenasterol, and stigmasterol increased significantly after dehiscence, while squalene and lupeol decreased after dehiscence; however, campesterol, clerosterol, and $\beta$-sitosterol exhibited no differences (Table 4).

Brassinolide (BL), the most biologically active brassinosteroid, is synthesized from campesterol [25]. Therefore, the presence of campesterol implies potential BL presence in ginseng seeds. However, since the amounts of BL in plants are often too low, it was analyzed from the unsaponifiable fraction of ginseng seed oil using GC-MS-selective ion monitoring. Bis-methaneboronate BL (BL-BMB) ions are observed at $\mathrm{m} / \mathrm{z} 528$, and their mass fragmentation is shown in Additional file 1: Fig. S3. Earlier studies have shown that fragment ions at $\mathrm{m} / \mathrm{z} 155,177$, and 374 are the most abundant, after analyzing BL-BMB using GC-EI-MS [26]. We selected the three fragment ions for use in the identification of BL-MB in ginseng seed. The peak areas of BL-BMB did not vary between the non-dehisced and the dehisced seeds, suggesting BL was not altered during dehiscence.

Plant steroid composition results showed that the phytosterols that increased following seed dehiscence belong to the stigmasterol biosynthesis pathway (Fig. 4), which also indicated that stigmasterol biosynthesis is activated in the course of warm stratification.

\section{Differential expression of enzymes based on real-time polymerase chain reaction (PCR)}

Numerous enzymes associated with the biosynthesis of plant steroids in ginseng have been reported [27, 28]. As illustrated in Fig. 4, squalene epoxidase (SE) converts squalene into 2,3-oxidosqualene. The 2,3-oxidosqualene transforms into three key compounds, cycloartenol, $\beta$-amyrin, and dammarenediol, via cycloartenol synthase (CAS), $\beta$-amyrin synthase ( $\beta$-AS), and dammarenediol synthase (DDS) activities, respectively. Cycloartenol is one of the precursors of phytosterols. Oleananetype triterpene saponins originate from $\beta$-amyrin. In addition, dammarenediol is an essential precursor of the dammaranetype triterpene saponins. Following the activation of the biosynthetic pathway by CAS, a significant amount of intermediate 24-methylenelophenol is formed. The biosynthetic pathway can induce divergence in phytosterols synthesis. When C4demethylase is activated, it becomes episterol, which is the precursor of brassinolide. Meanwhile, 24-ethylidenelophenol, the precursor of stigmasterol, is formed via the methylation of 24-methylenelophenol through activation by 24methylenesterol C-methyltransferase (SMT2).

To validate that the stigmasterol biosynthesis pathway is activated mainly during warm stratification, SE, CAS, $\beta$-AS, DDS, and SMT2 were compared between pre-dehiscence and post-dehiscence seeds. According to Qi et al. [29], 9-cisepoxycarotenoid dioxygenase (NCED1) is increased significantly after warm stratification, so that it is a positive control for seed dehiscence. According to real-time PCR results, SE, CAS, and SMT2 expression were increased significantly after seed dehiscence, while $\beta$-AS expression was not altered. In addition, DDS was undetectable both before and after seed dehiscing (Fig. 5).

\section{Discussion}

P. ginseng seeds typically exhibit morphophysiological dormancy, which implies that they require both morphological and physiological dormancy release before germination can occur, which tale place in the form of warm stratification, called dehiscence, followed by cold stratification. The dehiscence process is crucial for the cultivation of ginseng because it can 
take more than 18 months to germinate ginseng seeds if the dehiscence process does not take place appropriately when seeds are stored in the sand for 3 months at a temperature of $15-20^{\circ} \mathrm{C}[11,30]$. Therefore, a few studies have attempted to determine strategies of increasing ginseng seed dehiscence rates. For instance, Yang et al. [31] reported treatment with gibberellin $\left(\mathrm{GA}_{3}\right)$ enhances seed dehiscence rates when compared with that by warm stratification exclusively. In addition, increasing $\mathrm{GA}_{3}$ concentrations could also increase seed dehiscence rates [32,33]. Furthermore, the treatment of ginseng seeds with kinetin in combination with $\mathrm{GA}_{3}$ could increase dehiscence rate to $96.2 \%$, when compared with a rate of $90.6 \%$ in seeds exposed to warm stratification alone [34]. Although several studies have been conducted to find ways of increasing dehiscence rates in seeds, the underlying molecular mechanisms of seed dehiscence have remained unclear. In a previous study on American ginseng (Panax quinquefolius L.) seeds, 25,190 genes were compared through transcriptomic analysis based on the Kyoto Encyclopedia of Genes and Genomes (KEGG) pathway annotation in digital gene expression libraries. In addition, enrichment pathway analysis revealed pathways potentially associated with seed dormancy releases, such as steroid biosynthesis, flavonoid biosynthesis, flavone and flavonol biosynthesis, and starch and sucrose metabolism [29], and a transcriptomic analysis on Panax notoginseng (Burk) F.H. Chen seeds revealed 78 differentially expressed genes (DEGs) associated with seed dormancy, and among them, 15 DEGs were involved in abscisic acid (ABA) and $\mathrm{GA}_{3}$ pathways [35].

In the current study, by comparing metabolome of before and after dehisced $P$. ginseng seeds, we found that amino acids and sugars were significantly altered during the dehiscence. Freshly harvested ginseng seeds have an undeveloped embryo [30]. Therefore, during warm stratification, the alteration of amino acid and sugar concentrations, as well as composition, could be associated with embryogenesis of ginseng seed. Indeed, similar physiological phenomena, which are strongly associated with starch and protein accumulation, have been reported in Arabidopsis thaliana and Theobroma cacao L. seeds [36, 37]. Furthermore, the decrease in squalene concentrations following seed dehiscence was notable in the present study. In the triterpene biosynthetic pathway in ginseng, squalene is converted into 2,3-oxidosqualene by SE, and the 2,3oxidosqualene is a precursor of two different types of ginsenosides, dammarene type and oleanane type, in addition to phytosterols including brassinosteroids [38, 39].

Ginsenosides are some of the most pharmaceutically essential compounds in ginseng, and some studies have been conducted to increase their concentrations in $P$. ginseng roots, with a primary focus on squalene. Overexpression of the squalene synthase gene increased the levels of downstream enzymes, including SE, CAS, and $\beta$-AS, which, in turn, increased ginsenoside and phytosterol concentrations [40]. In addition, Sivakumar et al. [41] reported that exposing $P$. ginseng root to exogenous squalene increased phytosterol as well as ginsenoside concentrations. Therefore, the alteration of squalene concentrations in the present study suggested shifts in ginsenoside or phytosterol concentrations. Ginsenosides were not observed neither before nor after ginseng seed dehiscence using high-performance liquid chromatography-ultraviolet-visible spectroscopy (data not shown). However, one of the end-products of phytosterol biosynthesis, stigmasterol, increased significantly after ginseng seed dehiscence; conversely, the concentrations of other end-products, campesterol and brassinolide, were not altered. Phytosterols are primary components of the cell membrane, and they facilitate cell fluidity and permeability, similar to other sterols. Phytosterol synthesis is increased during seed germination to meet the demands for new membranes, and with seed maturity, the rate of increase declines gradually. The role of phytosterols in cellular proliferation and differentiation have also been elucidated. Although cholesterol alone restores growth by only $40-50 \%$ when compared to that in the control, with stigmasterol alone, it is possible to achieve full growth $[42,43]$.

Phytosterols can also regulate membrane-bound enzymes. For example, stigmasterol can stimulate proton pumps in cellular membranes; therefore, it participates in some signal transduction activities associated with seed development [4245]. Phytosterols are also precursors of some plant hormones, particularly brassinosteroids (BRs). It has already been reported that BRs regulate cell elongation and differentiation. Consequently, BRs control seed germination, development, and tolerance to numerous stress factors [46-48]. BRs perform "cross-talk" with key growth-regulating hormones, $\mathrm{GA}_{3}$, and 
ABA. BR binding into BRI1 (BRASSINOSTEROID INSENSITIVE 1) leads to heterodimerization with BAK1

(BRASSINOSTEROID INSENSITIVE 1-ASSOCIATED RECEPTOR KINASE 1), and the dimer prohibits BIN2 (BRASSINOSTEROID INSENSITIVE 2) the phosphorylation of BZR1/BES1 (BRASSINAZOLE RESISTANT 1/BRI1-EMSSUPPRESSOR 1). Activated BZR1/BES1 can promote $\mathrm{GA}_{3}$ biosynthesis, and $\mathrm{GA}_{3}$ degradation of DELLA proteins, and, in turn, the repression of BZR1/BES1 activity. It has also been reported that BR and ABA are negative regulators of each other. Increased $A B A$ levels upregulate BIN2, one of the primary negative regulators of $B R$ signaling, and $B R$ also inhibits $A B A$ activity through PP2C (PROTEIN PHOSPHATASE 2C) $[49,50]$. In a previous study, ABA levels increased during the dehiscence period in $P$. ginseng seeds, and after dehiscence, ABA levels decreased discernably, while gibberellic acid levels were not altered in the course of dehiscence, and increased after seed dehiscence [51]. The increased ABA levels could regulate $B R$ levels during the seed dehiscence period, leading to no change in BR after seed dehiscence.

The results of the present study demonstrated that metabolites are involved substantially in morphological growth changes during $P$. ginseng seed dehiscence. The results suggest that seed growth is the primary activity taking place under warm stratification, while other physiological changes, such as hormonal changes, are expected to take place later. For germination to occur in $P$. ginseng seed, warm stratification followed by cold stratification is required. Therefore, to further enhance our understanding of the mechanisms of $P$. ginseng seed germination, additional in-depth investigations are warranted to unravel the metabolic activities and alterations that unfold in the course of the cold stratification process.

\section{Conclusions}

The metabolomes of ginseng seeds before and after dehiscence were analyzed using gas GC-MS and compared to identify differentially expressed metabolites between the two groups. Sugar, amino acid profiles, and particularly squalene concentrations, were altered after seed dehiscence. Phytosterols, precursor metabolites of squalene that are associated with seed growth, increased after seed dehiscence. In addition, enzymes associated with phytosterol biosynthesis, SE, CAS, and SMT2, increased after seeds dehiscence. Nevertheless, other secondary metabolites, such as ginsenosides and brassinosteroids, were potentially not associated with the ginseng seed dehiscence. Overall, the results of the present study suggest that in the course of $P$. ginseng seed dehiscence, morphological activities, particularly growth, are dominant.

\section{Methods}

\section{Plant materials}

P. ginseng seeds used in the present study were harvested in July 2016 and were provided by Gyeonggi Eastern Ginseng Nonghyup (Incheon, Korea). Seeds were kept at $14^{\circ} \mathrm{C}$ for 16 weeks for seed coat dehiscence. Seeds were checked every week, and wholly dehisced seeds were stored at $-80^{\circ} \mathrm{C}$ until analyses.

\section{Chemical reagents}

Chloroform, methanol, water, methylene chloride, and methyl tert-butyl ether were purchased from JT Baker (Philipsburg, NJ, USA). Campesterol, $\beta$-sitosterol, clerosterol, $\Delta^{5}$-avenasterol, lupeol, and 24-methylenecycloartanol were obtained from ChemFaces Biochemical CO., Ltd. (Wuhan, Hubei, China). Benzoic acid (d5) was purchased from Cambridge Isotope Laboratory (Andover, MA, USA). All other chemical reagents were obtained from Sigma Aldrich (St. Louis, MO, USA).

\section{Untargeted metabolomics}

Seed coats were removed to prevent potential contamination. Afterward, the embryos and the endosperms were ground using a mortar and pestle under liquid nitrogen and lyophilized, and then $35 \mathrm{mg}$ of the samples were dissolved in $1 \mathrm{~mL}$ of a chloroform:methanol:water (2:5:2) mixture. Benzoic acid (d5) was spiked as an internal standard. The samples were vortexed and ultra-sonicated (Sonics \& Material, Inc., Newtown, CT, USA) for $30 \mathrm{~min}$ at $40^{\circ} \mathrm{C}$, followed by centrifugation at 
$16,000 \times g$ for $5 \mathrm{~min}$. The supernatants $(600 \mu \mathrm{L})$ were obtained for use in analyses, and the remaining $300 \mu \mathrm{L}$ supernatant were used as QC samples. The samples were dried under nitrogen purge at $50^{\circ} \mathrm{C}$. Thereafter, $100 \mu \mathrm{L}$ of anhydrous toluene was added, and the samples were dried again under nitrogen purge to eliminate all solvent, followed by derivatization. First, $100 \mu \mathrm{L}$ of methoxyamine hydrochloride in pyridine $(20 \mathrm{mg} / \mathrm{mL})$ was added, and samples were incubated for $90 \mathrm{~min}$ at $30^{\circ} \mathrm{C}$. Afterward, $100 \mu \mathrm{L}$ of $\mathrm{N}, \mathrm{O}$-bis(trimethylsilyl)trifluoroacetamide with trimethylchlorosilane (1\% TMCS) was added, and samples were incubated again for $15 \mathrm{~min}$ at $70^{\circ} \mathrm{C}$. The final $200 \mu \mathrm{L}$ of the samples were analyzed using GC-MS.

The extracted samples were analyzed using a gas chromatography-mass spectrometer (Shimadzu-QP2010, Kyoto, Japan) with a DB-5MS column ( $30 \mathrm{~m} \times 0.25 \mathrm{~mm}, 0.25 \mu \mathrm{m}$, Agilent, Santa Clara, CA, USA) to profile ginseng seed metabolites. The injection volume was $1 \mu \mathrm{L}$, and split mode with a ratio 5:1 was used as the injection mode. The injection temperature was $300^{\circ} \mathrm{C}$. Helium was used as a carrier gas with a flow rate of $1 \mathrm{~mL} / \mathrm{min}$. The initial oven temperature was maintained at $70^{\circ} \mathrm{C}$, increased to $150^{\circ} \mathrm{C}$ at a rate of $5^{\circ} \mathrm{C} / \mathrm{min}$, to $250^{\circ} \mathrm{C}$ at a rate of $8^{\circ} \mathrm{C} / \mathrm{min}$, to $300^{\circ} \mathrm{C}$ at a rate of $5^{\circ} \mathrm{C} / \mathrm{min}$, and then held at $300^{\circ} \mathrm{C}$ for $10 \mathrm{~min}$. The electron impact ionization method was used as a mass spectrometry detector with $70 \mathrm{eV}$ of electron energy, and mass data were collected in a scan mode at $30-600 \mathrm{~m} / \mathrm{z}$.

\section{Phytosterol content analysis}

Oil extraction and preparation of unsaponifiable fractions were performed according to previously described methods [24]. In brief, $5 \mathrm{~g}$ of ginseng seed power was boiled under reflux in $50 \mathrm{~mL}$ of methylene chloride for $2 \mathrm{~h}$ three times. After filtering, the extract was concentrated by rotary vacuum evaporation. One gram of the obtained oil was added into $20 \mathrm{~mL}$ of $1 \mathrm{M}$ $\mathrm{KOH}$ in methanol, and $1 \mathrm{~mL}$ of $0.1 \%(\mathrm{w} / \mathrm{v}) 5 \mathrm{a}$-cholestane in methyl tert-butyl ether added as an internal standard, and then stirred overnight. Subsequently, $40 \mathrm{~mL}$ of distilled water was added to dilute the mixture, and then extracted three times with $30 \mathrm{~mL}$ methyl tert-butyl ether. Afterward, $15 \mathrm{~mL}$ of $0.5 \mathrm{M} \mathrm{KOH}$ in methanol was added into the collected organic extract, and the solution was washed with $30 \mathrm{~mL}$ of distilled water until the $\mathrm{pH}$ became equivalent to the $\mathrm{pH}$ of the water, followed by the last wash with $15 \mathrm{~mL}$ of saturated sodium chloride solution. The solvent was eliminated by rotary vacuum evaporation.

Approximately $15 \mathrm{mg}$ of the unsaponifiable fraction was subjected to the above derivatization step and analyzed with the same GC-MS instrument. The initial oven temperature was held at $190^{\circ} \mathrm{C}$ for $2 \mathrm{~min}$, increased to $260^{\circ} \mathrm{C}$ at a rate of $5^{\circ} \mathrm{C} / \mathrm{min}$, to $275^{\circ} \mathrm{C}$ at a rate of $5^{\circ} \mathrm{C} / \mathrm{min}$, to $300^{\circ} \mathrm{C}$ at a rate of $5^{\circ} \mathrm{C} / \mathrm{min}$, and held at $300^{\circ} \mathrm{C}$ for $10 \mathrm{~min}$. The other conditions were similar to those described above.

\section{RNA extraction and real-time PCR analysis}

Total RNA was extracted from ginseng seeds using the RNeasy RNA Plant Mini Kit (Qiagen, Germany) according to the manufacturer's instructions. To eliminate genomic DNA, the samples were treated with RNase-Free DNase (Qiagen, Germany) before the final washing step. The quality and quantity of extracted RNA were measured using a NanoDrop ND1000 spectrophotometer (Thermo Fisher Scientific, Wilmington, USA). For real-time PCR analysis, cDNA was synthesized from $1 \mu \mathrm{g}$ of extracted RNA using M-MLV reverse transcriptase (Invitrogen, Carlsbad, CA, USA). The levels of expression of different genes were determined using an AB 7300 Real-time PCR system (Applied Biosystems, Foster City, CA, USA) with PowerUp SYBR green master mix (Applied Biosystems, Foster City, CA, USA) as the fluorescence reporter dye. The primer sequences of the target genes were SE (sense 5'-TCCTTTTTGGGTTCCTGCTC-3', anti-sense 5'-

GTATAAGCAAGAGCAGAGCCAGC-3'), cycloartenol synthase (CAS, sense 5'-GGGGAGTCTGCTTCACC-3', anti-sense 5'CTCCCCAACCACCAGAAG-3'), $\beta$-amyrin synthase ( $\beta$-AS, sense 5'-TATCCTGGACACCGAAAGAAGG-3', anti-sense 5'GTAGTATGTCTTTCCAGCTGCCG-3'), dammarenediol synthase (DDS, sense 5'-GCATACCGCCGTTGAGATTA-3', anti-sense 5'TAGTGTCAATCGTTCCGCTG-3'), 24-methylenesterol C-methyltransferase (SMT2, sense 5'-CTCTGCGAGGTGGTTCG-3', antisense 5'-GTCACCCACTCGTACGATAC-3'), 9-cis-epoxycarotenoid dioxygenase (NCED1, sense 5'-

CTTGTATGACTCCAGCGGAC-3', anti-sense 5'-TATTCACCATCCCTGCCTCT-3'), and glyceraldehyde 3-phosphate dehydrogenase (GAPDH, sense 5'-GTGGCCAGAGTGGCTTTG-3', anti-sense 5'-GACCGTGACTGGCTTCTCAC-3'). 


\section{Data preprocessing and compound identification}

Raw data were first converted to *.cdf files using GC-MS PostRun v.4.11 (Shimadzu). The *.cdf files were then converted into *.abf format files using the Reifycs Analysis Base File converter (Reifycs Inc., Tokyo, Japan). Afterward, the files were processed using MS-DIAL v.2.82), the universal and comprehensive software for metabolomics analysis [52]. The data collection parameters were as follows: Retention time begin of $5 \mathrm{~min}$, retention time end of $48.50 \mathrm{~min}$, mass range begin of $30 \mathrm{Da}$, and mass range end of $600 \mathrm{Da}$. For peak detection, linear weighted moving average with a level of 2 scans was applied for peak smoothing, average peak width was 5 scans, and minimum peak height was 2000 amplitude. For deconvolution parameters, sigma window value and electron ionization were 0.5 and 10 amplitudes, respectively. Peak alignment was processed using one QC file as a reference file, and other parameters were set as default.

The deconvoluted spectra were imported into MS Search (version 2.2) coupled with the NIST14 library for compound identification. Primarily, a match score of 700 was utilized as the threshold for putative identification. The retention index, the pattern of the peaks in the spectrum, and the reverse match score were also taken into consideration during the annotation process. The identities of differentially expressed metabolites, when applicable, were further confirmed by authentic commercial standards.

\section{Exploration of data analysis and visualization}

Processed spectral data were furthered log-transformed and scaled using by Pareto scaling method before actual analyses. PCA and heatmap analysis were mainly applied for data exploration and visualization. In addition, k-means clustering was used to obtained the first impression of the potential groupings of ginseng seeds before and after dehiscence.

\section{Statistical analysis}

In univariate analysis, a t-test was used to detect the differentially expressed metabolites between the non-dehisced and dehisced seed groups. In multivariate analysis, PLS-DA with cross-validation was conducted to develop the descriptive model of the separation between the two groups. All metabolites that were significantly different between two groups in ttest that also had a VIP score of 1.2 were selected for application in the development of a bio-signature of dehiscence. All metabolomics related analyses were complemented in MetaboAnalyst 4.0 [53]. Other statistical analyses were performed using IBM SPSS Statistics 25 (IBM Corp., Armonk, N.Y., USA). Continuous variables are presented as mean \pm standard deviation. A strict cut-off of adjusted P-value, FDR 0.001, was utilized to be the significance level for metabolomics experiments. FDR was adopted considering the multiple comparisons, which is a characteristic of high-dimensional analysis. The discriminant capacity of the bio-signature was validated using an independent set of samples based on ROC curve analysis using the PLS-DA algorithm. A P-value of 0.05 was applied for all other analyses unless otherwise stated.

\section{Functional enrichment analysis}

Pathway enrichment analysis of the differentially expressed metabolites was performed in MBRole v.2.0. Functional data on compounds were obtained from the KEGG database [54].

\section{Declarations}

\section{Ethics approval and consent to participate}

Not applicable

\section{Consent for publication}

Not applicable 
Availability of data and materials

All data generated or analyzed during this study are included in this published article and its supplementary information files.

Competing interests

The authors declare that they have no competing interests.

Funding

This research was supported by the Rural Development Administration of Korea (PJ01420102).

Authors' contributions

SWK, JHP, and JEM designed the study. JEM, NPL, JYH, SJK, and NHA performed the chemical and biological experiments, and analyzed and interpreted the data. SWK and JHP interpreted the data. JEM and NPL wrote the manuscript. All authors critically revised the manuscript and approved the final contents.

Acknowledgments

Not applicable.

\section{References}

1. Lee DC, Lau AS: Effects of Panax ginseng on tumor necrosis factor-a-mediated inflammation: a mini-review. Molecules 2011, 16(4):2802-2816.

2. Shergis JL, Zhang AL, Zhou W, Xue CC: Panax ginseng in randomised controlled trials: a systematic review. Phytotherapy Research 2013, 27(7):949-965.

3. Shin HR, Kim JY, Yun TK, Morgan G, Vainio H: The cancer-preventive potential of Panax ginseng: a review of human and experimental evidence. Cancer Causes \& Control 2000, 11(6):565-576.

4. Vogler B, Pittler M, Ernst E: The efficacy of ginseng. A systematic review of randomised clinical trials. European Journal of Clinical Pharmacology 1999, 55(8):567-575.

5. Reay JL, Kennedy DO, Scholey AB: Single doses of Panax ginseng (G115) reduce blood glucose levels and improve cognitive performance during sustained mental activity. Journal of Psychopharmacology 2005, 19(4):357-365.

6. Reay JL, Kennedy DO, Scholey AB: Effects of Panax ginseng, consumed with and without glucose, on blood glucose levels and cognitive performance during sustained 'mentally demanding'tasks. Journal of Psychopharmacology 2006, 20(6):771-781.

7. Kang S, Min H: Ginseng, the'immunity boost': the effects of Panax ginseng on immune system. Journal of ginseng research 2012, 36(4):354.

8. Kim SW, Gupta R, Lee SH, Min CW, Agrawal GK, Rakwal R, Kim JB, Jo IH, Park S-Y, Kim JK et al: An Integrated Biochemical, Proteomics, and Metabolomics Approach for Supporting Medicinal Value of Panax ginseng Fruits. Frontiers in Plant Science 2016, 7(994).

9. Baskin JM, Baskin CC: A classification system for seed dormancy. Seed science research 2004, 14(1):1-16.

10. Lee J-S, Lee S-S, Lee J-H, Ahn I-O: Effect of seed size and cultivars on the ratio of seed coat dehiscence and seedling performance in Panax ginseng. Journal of Ginseng Research 2008, 32(3):257-263.

11. Kwon W, Lee J, Lee M: Optimum chilling terms for germination of the dehisced ginseng (Panax ginseng CA Meyer) seed. J Ginseng Res 2001, 25(4):167-170. 
12. Patti GJ, Yanes O, Siuzdak G: Innovation: Metabolomics: the apogee of the omics trilogy. Nature reviews: Molecular cell biology 2012, 13(4):263.

13. Weckwerth W: Metabolomics in systems biology. Annual review of plant biology 2003, 54(1):669-689.

14. Xia J, Broadhurst DI, Wilson M, Wishart DS: Translational biomarker discovery in clinical metabolomics: an introductory tutorial. Metabolomics 2013, 9(2):280-299.

15. Styczynski MP, Moxley JF, Tong LV, Walther JL, Jensen KL, Stephanopoulos GN: Systematic identification of conserved metabolites in GC/MS data for metabolomics and biomarker discovery. Analytical chemistry 2007, 79(3):966-973.

16. Alonso A, Marsal S, Julià A: Analytical methods in untargeted metabolomics: state of the art in 2015. Frontiers in bioengineering and biotechnology 2015, 3:23.

17. Nguyen HT, Lee D-K, Choi Y-G, Min J-E, Yoon SJ, Yu Y-H, Lim J, Lee J, Kwon SW, Park JH: A 1H NMR-based metabolomics approach to evaluate the geographical authenticity of herbal medicine and its application in building a model effectively assessing the mixing proportion of intentional admixtures: a case study of Panax ginseng: metabolomics for the authenticity of herbal medicine. Journal of pharmaceutical and biomedical analysis 2016, 124:120-128.

18. Kim J, Jung Y, Song B, Bong Y-S, Lee K-S, Hwang G-S: Discrimination of cabbage (Brassica rapa ssp. pekinensis) cultivars grown in different geographical areas using 1H NMR-based metabolomics. Food chemistry 2013, 137(14):68-75.

19. Quanbeck S, Brachova L, Campbell A, Guan X, Perera A, He K, Rhee S, Bais P, Dickerson J, Dixon P et al: Metabolomics as a Hypothesis-Generating Functional Genomics Tool for the Annotation of Arabidopsis thaliana Genes of "Unknown Function". Frontiers in Plant Science 2012, 3(15).

20. Emwas A-HM: The strengths and weaknesses of NMR spectroscopy and mass spectrometry with particular focus on metabolomics research. In: Metabonomics. Springer; 2015: 161-193.

21. Jayakodi M, Lee S-C, Lee YS, Park H-S, Kim N-H, Jang W, Lee HO, Joh HJ, Yang T-J: Comprehensive analysis of Panax ginseng root transcriptomes. BMC Plant Biology 2015, 15(1):138.

22. Li X-J, Yang J-L, Hao B, Lu Y-C, Qian Z-L, Li Y, Ye S, Tang J-R, Chen M, Long G-Q et al: Comparative transcriptome and metabolome analyses provide new insights into the molecular mechanisms underlying taproot thickening in Panax notoginseng. BMC Plant Biology 2019, 19(1):451.

23. Fan H, Li K, Yao F, Sun L, Liu Y: Comparative transcriptome analyses on terpenoids metabolism in field- and mountaincultivated ginseng roots. BMC Plant Biology 2019, 19(1):82.

24. Beveridge TH, Li TS, Drover JC: Phytosterol content in American ginseng seed oil. Journal of Agricultural and Food Chemistry 2002, 50(4):744-750.

25. Noguchi T, Fujioka S, Choe S, Takatsuto S, Tax FE, Yoshida S, Feldmann KA: Biosynthetic pathways of brassinolide in Arabidopsis. Plant Physiology 2000, 124(1):201-210.

26. Takatsuto S: Brassinosteroids: distribution in plants, bioassays and microanalysts by gas chromatography-mass spectrometry. Journal of Chromatography A 1994, 658(1):3-15.

27. Li J, Wang C, Han X, Qi W, Chen Y, Wang T, Zheng Y, Zhao X: Transcriptome analysis to identify the putative biosynthesis and transport genes associated with the medicinal components of Achyranthes bidentata BI. Frontiers in plant science 2016, 7:1860.

28. Kim Y-K, Kim YB, Uddin MR, Lee S, Kim S-U, Park SU: Enhanced triterpene accumulation in Panax ginseng hairy roots overexpressing mevalonate-5-pyrophosphate decarboxylase and farnesyl pyrophosphate synthase. ACS synthetic biology 2014, 3(10):773-779.

29. Qi J, Sun P, Liao D, Sun T, Zhu J, Li X: Transcriptomic analysis of American ginseng seeds during the dormancy release process by RNA-Seq. PloS one 2015, 10(3):e0118558. 
30. Kim M-J, Shim C-K, Kim Y-K, Hong S-J, Park J-H, Han E-J, Kim S-C: Enhancement of Seed Dehiscence by Seed Treatment with Talaromyces flavus GG01 and GG04 in Ginseng (Panax ginseng). The plant pathology journal 2017, 33(1):1.

31. Yang D, Cheon S, Lee S, Yang D, Kim H: The effects of various dehiscence materials, growth regulators and fungicides on the ginseng seed. Korean J Ginseng Sci 1982, 6:56-66.

32. Kim YC, Kim YB, Park HW, Bang KH, Kim JU, Jo IH, Kim KH, Song BH, Kim DH: Optimal harvesting time of ginseng seeds and effect of gibberellic acid (GA 3) treatment for improving stratification rate of ginseng (Panax ginseng CA Meyer) seeds. Korean Journal of Medicinal Crop Science 2014, 22(6):423-428.

33. Lee JW, NIHHS R, Kim Y, NIHHS R, Kim J, NIHHS R, Jo I, NIHHS R, Kim K, NIHHS R: Effects of gibberellic acid and alternating temperature on breaking seed dormancy of Panax ginseng CA Meyer. Korean Journal of Medicinal Crop Science 2016.

34. Lee J-W, Jo I-H, Kim J-U, Hong C-E, Kim Y-C, Kim D-H, Park Y-D: Improvement of seed dehiscence and germination in ginseng by stratification, gibberellin, and/or kinetin treatments. Horticulture, Environment, and Biotechnology 2018, 59(3):293-301.

35. Yang K, Yang L, Fan W, Long GQ, Xie SQ, Meng ZG, Zhang GH, Yang SC, Chen JW: Illumina-based transcriptomic analysis on recalcitrant seeds of Panax notoginseng for the dormancy release during the after-ripening process. Physiologia plantarum 2018.

36. Alemanno L, Berthouly M, Michaux-Ferriere N: A comparison between Theobroma cacao L. zygotic embryogenesis and somatic embryogenesis from floral explants. In Vitro Cellular \& Developmental Biology-Plant 1997, 33(3):163-172.

37. Baud S, Boutin J-P, Miquel M, Lepiniec L, Rochat C: An integrated overview of seed development in Arabidopsis thaliana ecotype WS. Plant Physiology and Biochemistry 2002, 40(2):151-160.

38. Choi D-W, Jung J, Im Ha Y, Park H-W, In DS, Chung H-J, Liu JR: Analysis of transcripts in methyl jasmonate-treated ginseng hairy roots to identify genes involved in the biosynthesis of ginsenosides and other secondary metabolites. Plant cell reports 2005, 23(8):557-566.

39. Kim Y-S, Han J-Y, Lim S, Choi Y-E: Ginseng metabolic engineering: regulation of genes related to ginsenoside biosynthesis. Journal of Medicinal Plants Research 2009, 3(13):1270-1276.

40. Lee M-H, Jeong J-H, Seo J-W, Shin C-G, Kim Y-S, In J-G, Yang D-C, Yi J-S, Choi Y-E: Enhanced triterpene and phytosterol biosynthesis in Panax ginseng overexpressing squalene synthase gene. Plant and Cell Physiology 2004, 45(8):976984.

41. Sivakumar G, Yu K, Paek K: Enhanced production of bioactive ginsenosides from adventitious roots of Panax ginseng in bioreactor culture. J Hortic Sci Biotechnol 2006, 81(3):549-552.

42. Hartmann M-A: Plant sterols and the membrane environment. Trends in plant science 1998, 3(5):170-175.

43. Schaller $\mathrm{H}$ : The role of sterols in plant growth and development. Progress in lipid research 2003, 42(3):163-175.

44. Zhou W, Branch WD, Gilliam L, Marshall JA: Phytosterol Composition of Arachis hypogaea Seeds from Different Maturity Classes. Molecules 2018, 24(1):106.

45. Ferrer A, Altabella T, Arró M, Boronat A: Emerging roles for conjugated sterols in plants. Progress in lipid research 2017, 67:27-37.

46. Clouse SD: Brassinosteroid signal transduction: from receptor kinase activation to transcriptional networks regulating plant development. The Plant Cell 2011, 23(4):1219-1230.

47. Divi UK, Krishna P: Brassinosteroid: a biotechnological target for enhancing crop yield and stress tolerance. New biotechnology 2009, 26(3-4):131-136.

48. Vriet C, Russinova E, Reuzeau C: Boosting crop yields with plant steroids. The Plant Cell 2012, 24(3):842-857.

49. Peres ALGL, Soares JS, Tavares RG, Righetto G, Zullo MAT, Mandava NB, Menossi M: Brassinosteroids, the Sixth Class of Phytohormones: A Molecular View from the Discovery to Hormonal Interactions in Plant Development and Stress 
Adaptation. International Journal of Molecular Sciences 2019, 20(2):331.

50. Planas-Riverola A, Gupta A, Betegón-Putze I, Bosch N, lbañes $M$, Caño-Delgado Al: Brassinosteroid signaling in plant development and adaptation to stress. Development 2019, 146(5):dev151894.

51. Kim Y-H, Ahn I-O, Khan AL, Kamran M, Waqas M, Lee J-S, Kim D-H, Jang S-W, Lee I-J: Regulation of endogenous gibberellins and abscisic acid levels during different seed collection periods in Panax ginseng. Horticulture, Environment, and Biotechnology 2014, 55(3):166-174.

52. Tsugawa H, Cajka T, Kind T, Ma Y, Higgins B, Ikeda K, Kanazawa M, VanderGheynst J, Fiehn O, Arita M: MS-DIAL: dataindependent MS/MS deconvolution for comprehensive metabolome analysis. Nature methods 2015, 12(6):523.

53. Chong J, Soufan O, Li C, Caraus I, Li S, Bourque G, Wishart DS, Xia J: MetaboAnalyst 4.0: towards more transparent and integrative metabolomics analysis. Nucleic Acids Res 2018, 46(W1):W486-w494.

54. Lopez-Ibanez J, Pazos F, Chagoyen M: MBROLE 2.0-functional enrichment of chemical compounds. Nucleic Acids Res 2016, 44(W1):W201-204.

\section{Tables}

Table 1 Differentially expressed metabolites between before ginseng seed dehiscence and after ginseng seed dehiscence.

\begin{tabular}{|lll|}
\hline Compound name & Retention time (min) & Changes after dehisced \\
\hline I-valine & 6.66 & Increased \\
\hline d/I-2-aminobutyric acid & 8.55 & Increased \\
\hline I-leucine & 11.07 & Decreased \\
\hline I-isoleucine & 11.6 & Decreased \\
\hline I-serine & 13.37 & Increased \\
\hline I-threonine & 13.97 & Decreased \\
\hline Pentanedioic acid & 14.58 & Decreased \\
\hline I-5-oxoproline & 17.3 & Increased \\
\hline I-asparagine & 18.91 & Increased \\
\hline I-phenylalanine & 19.32 & Increased \\
\hline Tyrosine & 23.42 & Increased \\
\hline d-trehalose & 33.33 & Decreased \\
\hline myo-Inositol & 33.94 & Decreased \\
\hline Squalene & 34.39 & Decreased \\
\hline Galactinol & 36.18 & Increased \\
\hline
\end{tabular}

Table 2. Metabolic pathways involving identified metabolites predicted as dehiscence biomarkers 


\begin{tabular}{|c|c|c|c|c|c|c|c|c|c|}
\hline & $\begin{array}{l}\text { I- } \\
\text { valine }\end{array}$ & leucine & $\begin{array}{l}\text { I- } \\
\text { isoleucine }\end{array}$ & $\begin{array}{l}\text { I- } \\
\text { serine }\end{array}$ & $\begin{array}{l}\text { I- } \\
\text { threonine }\end{array}$ & $\begin{array}{l}\text { I- } \\
\text { asparagine }\end{array}$ & Tyrosine & $\begin{array}{l}\text { myo- } \\
\text { Inositol }\end{array}$ & Squalene \\
\hline $\begin{array}{l}\text { Aminoacyl- } \\
\text { tRNA } \\
\text { biosynthesis }\end{array}$ & 0 & 0 & 0 & 0 & 0 & 0 & 0 & - & - \\
\hline $\begin{array}{l}\text { Valine, } \\
\text { leucine, and } \\
\text { isoleucine } \\
\text { biosynthesis }\end{array}$ & 0 & 0 & 0 & - & 0 & - & - & - & - \\
\hline $\begin{array}{l}\mathrm{ABC} \\
\text { transporters }\end{array}$ & 0 & 0 & 0 & 0 & 0 & - & - & - & - \\
\hline $\begin{array}{l}\text { Biosynthesis } \\
\text { of secondary } \\
\text { metabolites }\end{array}$ & 0 & 0 & 0 & 0 & 0 & 0 & 0 & 0 & 0 \\
\hline $\begin{array}{l}\text { Glucosinolate } \\
\text { biosynthesis }\end{array}$ & 0 & 0 & 0 & - & - & - & 0 & - & - \\
\hline $\begin{array}{l}\text { Valine, } \\
\text { leucine, and } \\
\text { isoleucine } \\
\text { degradation }\end{array}$ & 0 & 0 & 0 & - & - & - & - & - & - \\
\hline $\begin{array}{l}\text { Cyanoamino } \\
\text { acid } \\
\text { metabolism }\end{array}$ & - & - & - & 0 & - & 0 & - & - & - \\
\hline $\begin{array}{l}\text { Metabolic } \\
\text { pathways }\end{array}$ & 0 & 0 & 0 & 0 & 0 & 0 & 0 & 0 & 0 \\
\hline
\end{tabular}

* The pathway related to d/I-2-aminobutyric acid, pentanedioic acid, I-5-oxoproline, I-phenylalanine, d-trehalose, and galactinol was not identified.

Table 3. Fragmentation ions for identification of phytosterols in Panax ginseng 


\begin{tabular}{|c|c|c|c|c|c|c|c|}
\hline \multirow[t]{2}{*}{ Peak } & \multirow[t]{2}{*}{ Compound } & \multicolumn{6}{|c|}{ Fragmentation ions, $m / z(\mathrm{RI})^{\mathrm{a}}$} \\
\hline & & $M+$ & $\begin{array}{l}M- \\
15\end{array}$ & $\begin{array}{l}\text { M- } \\
90\end{array}$ & $\begin{array}{l}M- \\
105\end{array}$ & $\begin{array}{l}M- \\
129\end{array}$ & others \\
\hline 1 & Squalene & $\begin{array}{l}410 \\
(1)\end{array}$ & & & & & $\begin{array}{l}367(1), 341(2), 231(1), 203(2), 189(2), 149 \\
(11), 137(18), 136(20), 123(14), 121(19), 109 \\
(13), 107(12), 95(27), 93(16), 82(11), 81(93), \\
69^{b}\end{array}$ \\
\hline 2 & 2,3-Oxidosqualene & $\begin{array}{l}426 \\
(1)\end{array}$ & & & & & $\begin{array}{l}357(1), 231(1), 203(3), 191(2), 149(9), 135 \\
(22), 133(7), 123(14), 121(17), 109(18), 107 \\
(19), 95(28), 93(25), 81(90), 71(50), 69 \mathrm{~b}\end{array}$ \\
\hline 3 & Campesterol & $\begin{array}{l}472 \\
(19)\end{array}$ & $\begin{array}{l}457 \\
(5)\end{array}$ & $\begin{array}{l}382 \\
(47)\end{array}$ & $\begin{array}{l}367 \\
(23)\end{array}$ & $\begin{array}{l}343 \\
(47)\end{array}$ & $315(3), 255(14), 145(31), 129^{b}$ \\
\hline 4 & Stigmasterol & $\begin{array}{l}484 \\
(20)\end{array}$ & $\begin{array}{l}469 \\
(3)\end{array}$ & $\begin{array}{l}394 \\
(23)\end{array}$ & $\begin{array}{l}379 \\
(8)\end{array}$ & $\begin{array}{l}355 \\
(8)\end{array}$ & $\begin{array}{l}343(4), 255(26), 213(8), 145(19), 133(21) \\
129(53), 97(20), 95(19), 83^{\text {b }}\end{array}$ \\
\hline 5 & Clerosterol & $\begin{array}{l}484 \\
(33)\end{array}$ & $\begin{array}{l}469 \\
(8)\end{array}$ & $\begin{array}{l}394 \\
(25)\end{array}$ & $\begin{array}{l}379 \\
(11)\end{array}$ & $\begin{array}{l}355 \\
(16)\end{array}$ & $\begin{array}{l}386(6), 379(11), 355(16), 296(11), 255(13), \\
213(13), 139(30), 129(88), 119(38), 107(35), \\
81(48), 55^{b}\end{array}$ \\
\hline 6 & $\beta$-Sitosterol & $\begin{array}{l}486 \\
(25)\end{array}$ & $\begin{array}{l}471 \\
(7)\end{array}$ & $\begin{array}{l}396 \\
(52)\end{array}$ & $\begin{array}{l}381 \\
(20)\end{array}$ & $\begin{array}{l}357 \\
(47)\end{array}$ & $\begin{array}{l}394(25), 255(15), 213(9), 145(29), 129^{b}, 95 \\
(40), 73(50)\end{array}$ \\
\hline 7 & $\Delta^{5}$-Avenasterol & $\begin{array}{l}484 \\
(6)\end{array}$ & $\begin{array}{l}469 \\
(5)\end{array}$ & $\begin{array}{l}394 \\
(3)\end{array}$ & $\begin{array}{l}379 \\
(5)\end{array}$ & $\begin{array}{l}355 \\
(5)\end{array}$ & $\begin{array}{l}386(79), 296(68), 281(45), 257(24), 255(14), \\
129^{\text {b }}, 95(47), 55(94)\end{array}$ \\
\hline 8 & $\Delta^{5,24(25)}$-Stigmasterol & $\begin{array}{l}484 \\
(11)\end{array}$ & $\begin{array}{l}469 \\
(5)\end{array}$ & $\begin{array}{l}394 \\
(6)\end{array}$ & $\begin{array}{l}379 \\
(7)\end{array}$ & $\begin{array}{l}355 \\
(4)\end{array}$ & $\begin{array}{l}386(43), 343(9), 296(39), 281(26), 257(14), \\
255(11), 253(17), 129(73), 121(26), 119(40), \\
107(32), 105(29), 97(68), 95(37), 93(31), 81 \\
(40), 75(42), 73(72), 69(67), 55^{b}\end{array}$ \\
\hline 9 & Lupeol & $\begin{array}{l}498 \\
(16)\end{array}$ & $\begin{array}{l}483 \\
(7)\end{array}$ & $\begin{array}{l}408 \\
(11)\end{array}$ & $\begin{array}{l}393 \\
(13)\end{array}$ & $\begin{array}{l}369 \\
(13)\end{array}$ & $\begin{array}{l}279(12), 218(31), 203(41), 190(53), 189(75), \\
136(45), 135(64), 123(45), 121(64), 109(94), \\
107(65), 95(92), 93(61), 81(71), 75(75), 73 \text { b }\end{array}$ \\
\hline 10 & $\Delta^{7}$-Avenasterol & $\begin{array}{l}484 \\
(2)\end{array}$ & $\begin{array}{l}469 \\
(6)\end{array}$ & $\begin{array}{l}394 \\
(1)\end{array}$ & $\begin{array}{l}379 \\
(4)\end{array}$ & & $\begin{array}{l}386(41), 371(6), 343^{\text {b }}, 296(7), 281(9), 253 \\
(27), 145(18), 119(17)\end{array}$ \\
\hline 11 & $\begin{array}{l}\text { 24- } \\
\text { Methylenecycloartanol }\end{array}$ & $\begin{array}{l}512 \\
(1)\end{array}$ & $\begin{array}{l}497 \\
(3)\end{array}$ & $\begin{array}{l}422 \\
(40)\end{array}$ & $\begin{array}{l}407 \\
(38)\end{array}$ & & $\begin{array}{l}379(54), 353(15), 300(9), 147(41), 135(61), \\
121(55), 109(60), 107(62), 105(44), 95(83), \\
81(58), 73^{\text {b }}\end{array}$ \\
\hline 12 & Citrostadienol & $\begin{array}{l}498 \\
(2)\end{array}$ & $\begin{array}{l}483 \\
(4)\end{array}$ & $\begin{array}{l}408 \\
(2)\end{array}$ & $\begin{array}{l}393 \\
(6)\end{array}$ & & $400(51), 357^{\mathrm{b}}, 267(27), 145(21), 133(22)$ \\
\hline
\end{tabular}

${ }^{a}$ Relative intensity compared to base peak (percent); ${ }^{b}$ Base peak (relative intensity $=100 \%$ )

Table 4. Phytosterol contents before and after Panax ginseng seed dehiscing 


\begin{tabular}{|c|c|c|c|}
\hline \multirow[t]{2}{*}{ Peak } & \multirow[t]{2}{*}{ Compound } & \multicolumn{2}{|c|}{ Contents (Milligrams per $100 \mathrm{~g}$ of seed oil, $\mathrm{n}=3$ ) } \\
\hline & & Before dehisced & After dehisced \\
\hline 1 & Squalene $e^{a}$ & $166.05 \pm 11.15$ & $120.19 \pm 21.08$ \\
\hline 2 & 2,3-Oxidosqualene ${ }^{a}$ & $362.75 \pm 32.92$ & $616.45 \pm 47.45$ \\
\hline 3 & Campesterol & $1.85 \pm 0.70$ & $2.05 \pm 0.33$ \\
\hline 4 & Stigmasterol ${ }^{\mathrm{a}}$ & $25.58 \pm 4.00$ & $48.91 \pm 0.64$ \\
\hline 5 & Clerosterol & $1.37 \pm 0.40$ & $1.29 \pm 0.42$ \\
\hline 6 & $\beta$-Sitosterol & $55.22 \pm 2.25$ & $58.72 \pm 6.36$ \\
\hline 7 & $\Delta^{5}$-Avenasterol ${ }^{\mathrm{a}}$ & $138.52 \pm 6.68$ & $170.71 \pm 7.98$ \\
\hline 8 & $\Delta^{5,24(25)}$-Stigmasterol & - & - \\
\hline 9 & Lupeol $^{\mathrm{a}}$ & $14.86 \pm 0.12$ & $8.71 \pm 0.64$ \\
\hline 10 & $\Delta^{7}$-Avenasterol & - & - \\
\hline 11 & 24-Methylenecycloartanol & $9.26 \pm 1.02$ & $10.20 \pm 0.92$ \\
\hline 12 & Citrostadienol & - & - \\
\hline
\end{tabular}

a Statistically significant difference before and after dehiscing $(P$-value $<0.05)$

\section{Figures}



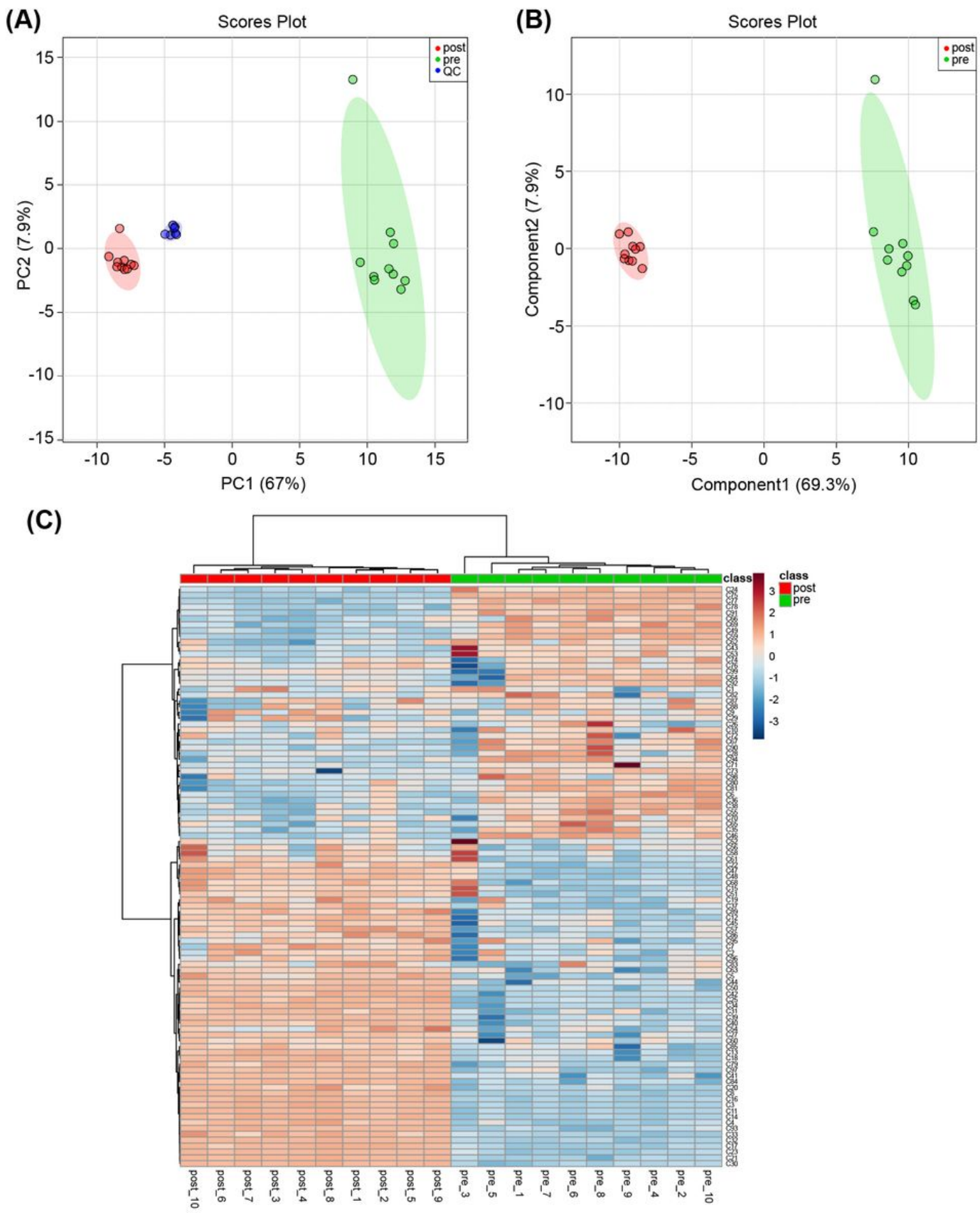

\section{Figure 1}

Score plot of principal component analysis (PCA) (a), score plot of partial least squares discriminant analysis (PLS-DA) (b), and heatmap visualization (c) of before seed dehiscing and after seed dehiscing. Red represents after dehiscing, green represents before dehiscing, and blue represents quality control (QC) samples. In the heatmap, "Cn" denotes the detected peaks. 
(A)

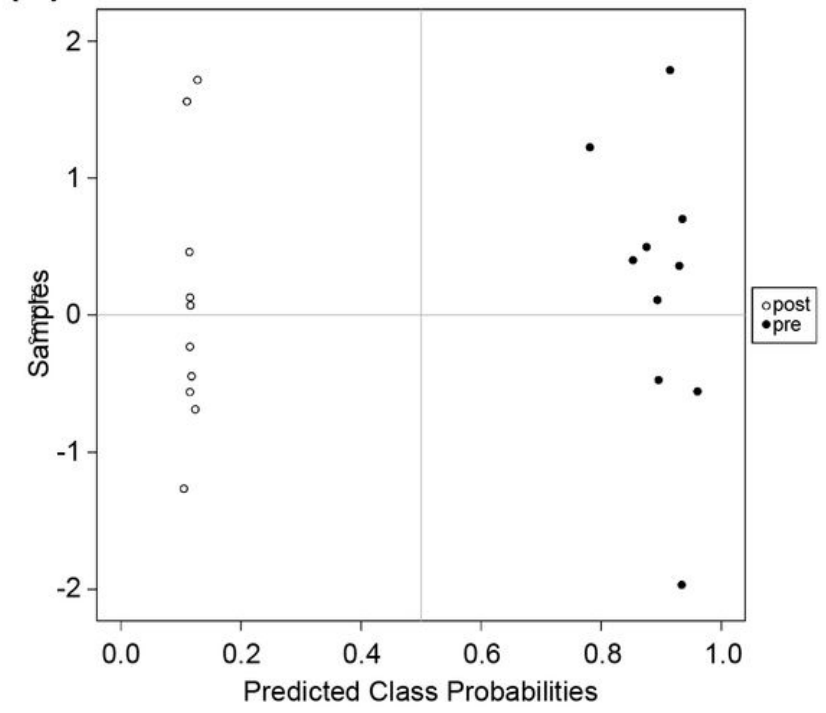

(B)

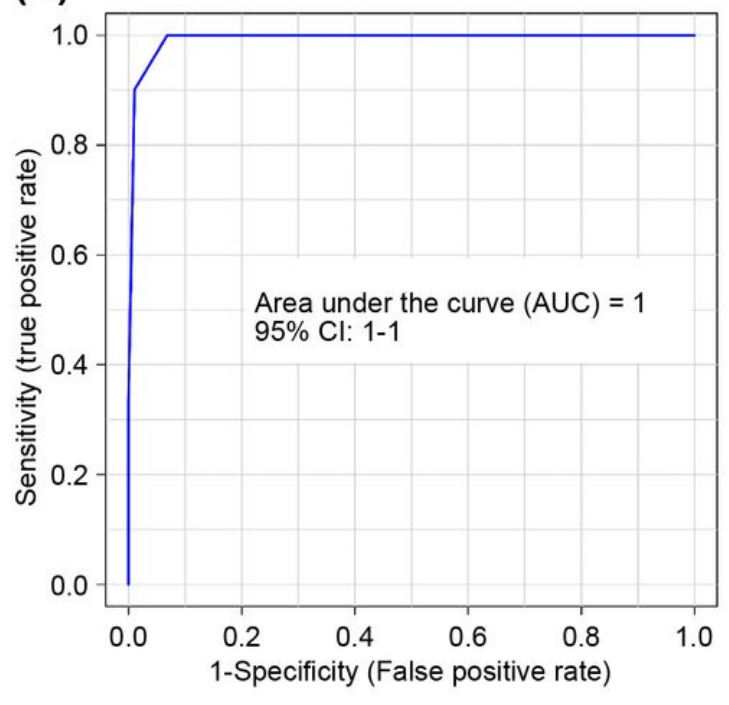

(C)

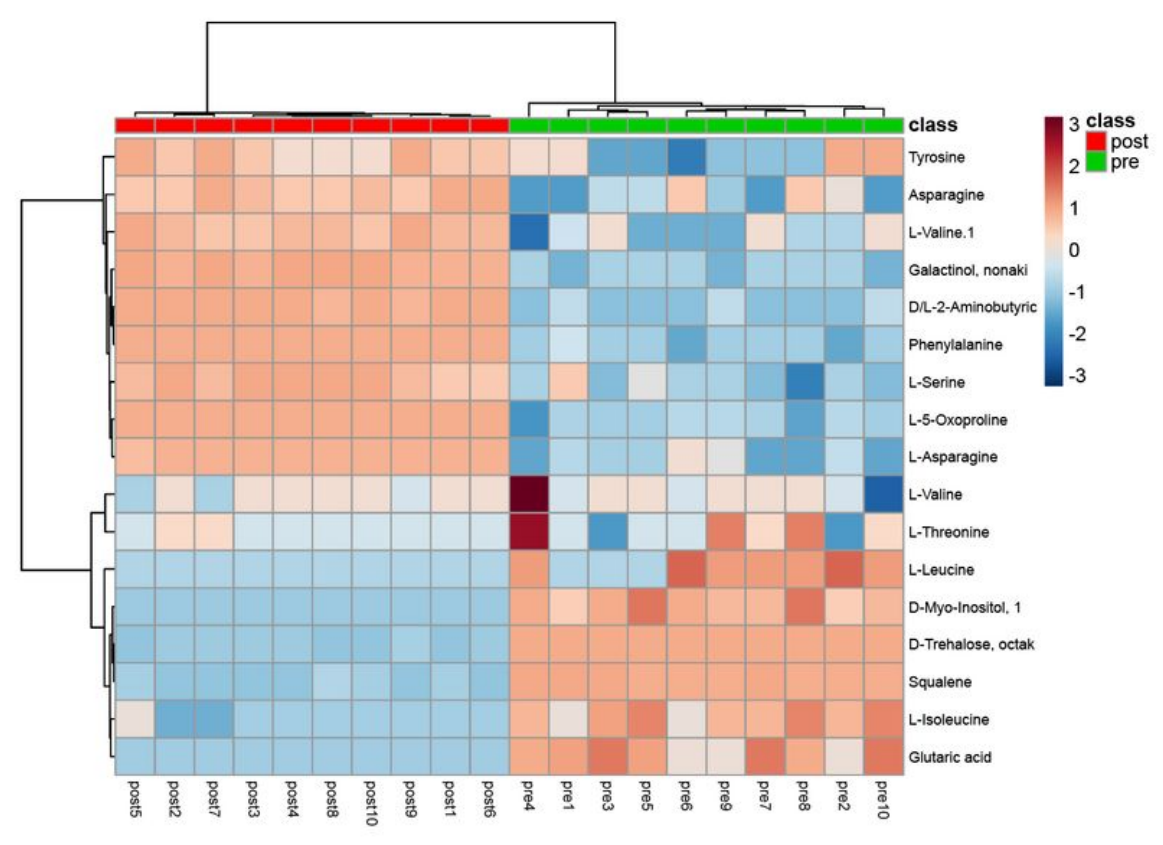

Figure 2

Specificity and sensitivity of identified metabolites as biomarkers. Class distribution (a), receiver operating characteristic (ROC) curve analysis (b), and heatmap analysis (c) revealed distinction between before and after seed dehiscing. 


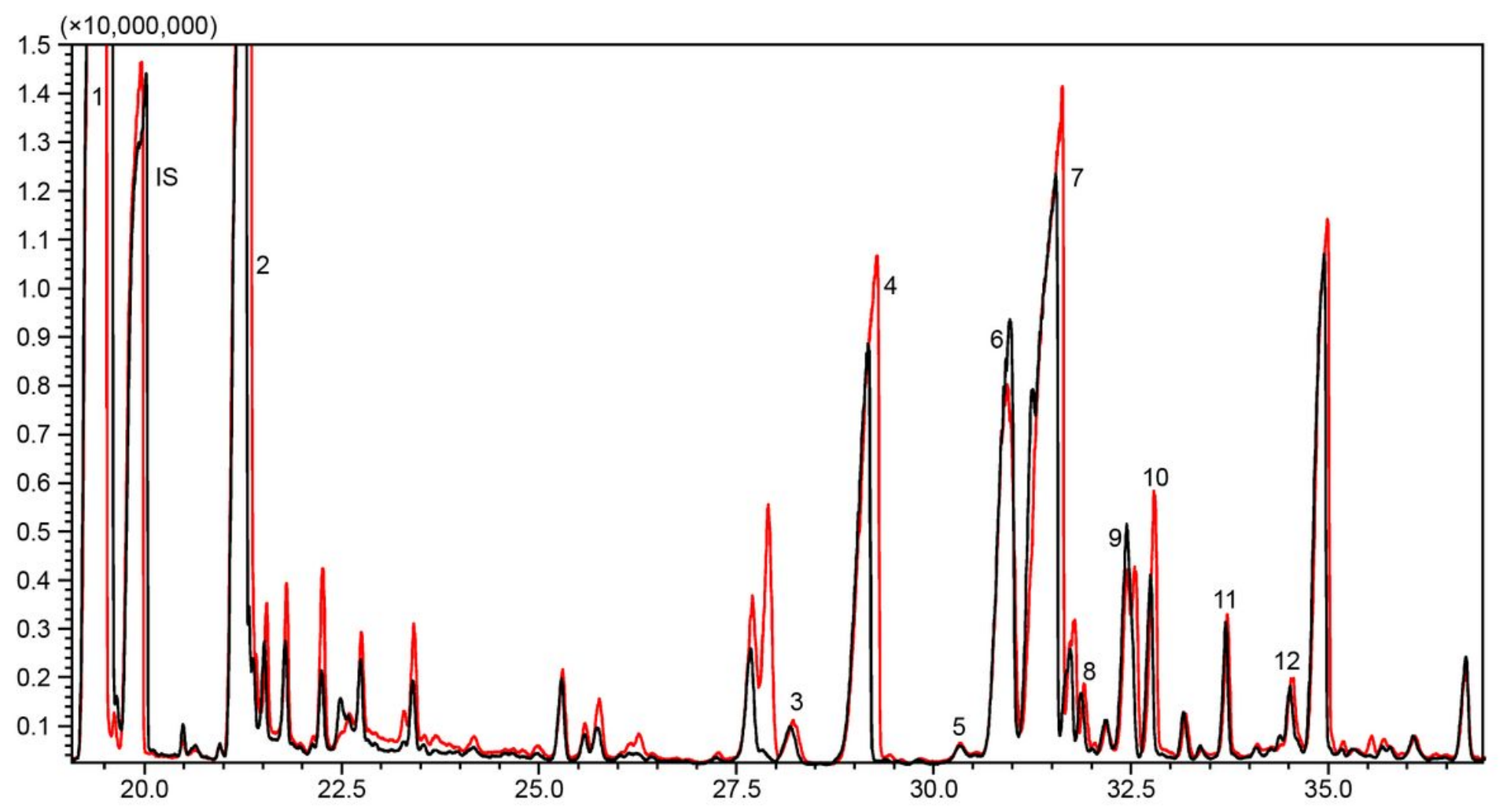

Figure 3

Overlaid gas chromatograph-mass spectrometry (GC-MS) chromatogram of phytosterol fractions before (black) and after (red) Panax ginseng seed dehiscing. 5a-Cholestane was used as the internal standard (IS). Identified peaks are listed in Table 3.

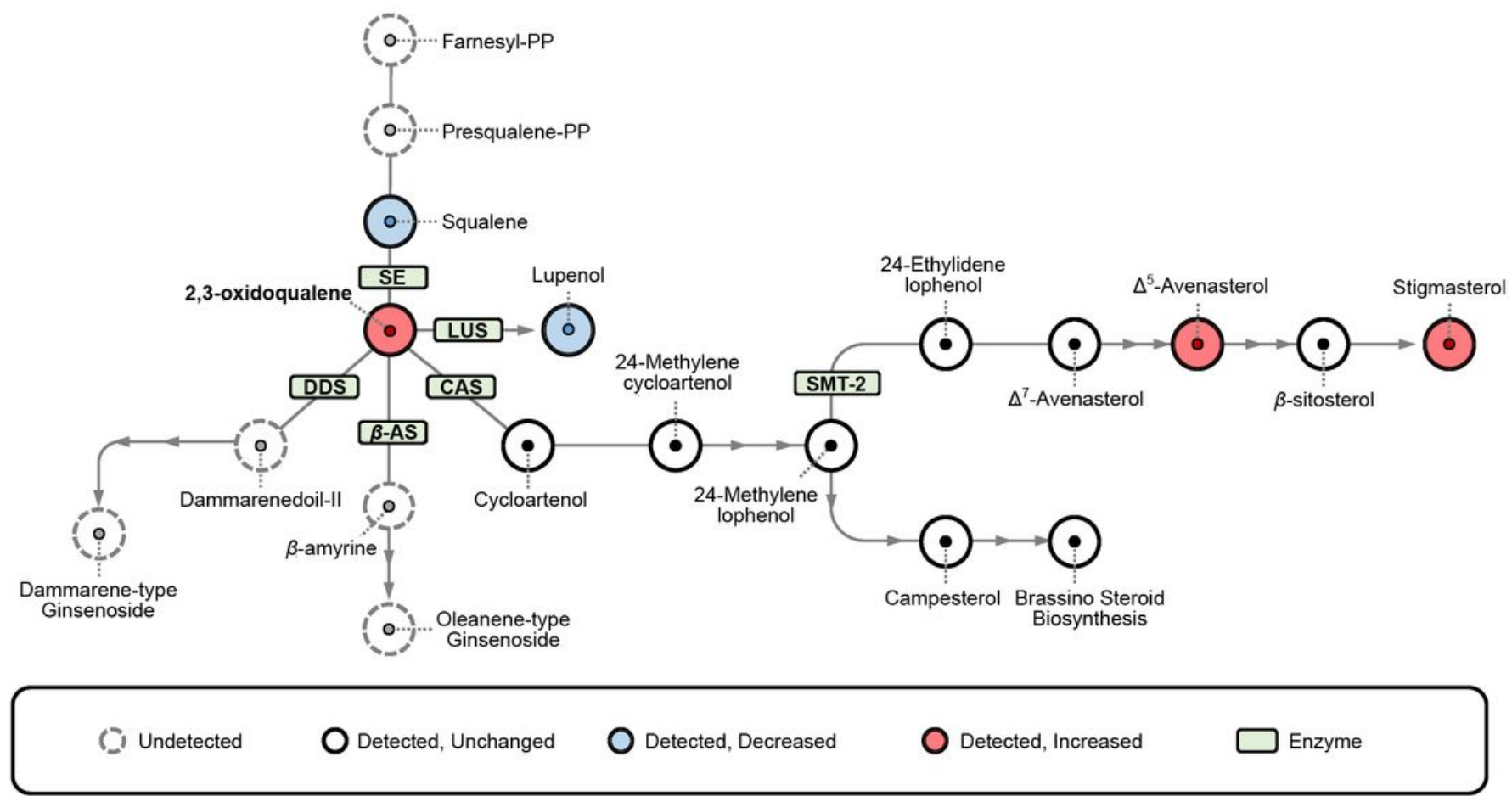


Figure 4

Trends observed in phytosterol biosynthesis pathways in the present study. Red phytosterols increased, while blue phytosterols decreased after seed dehiscing.

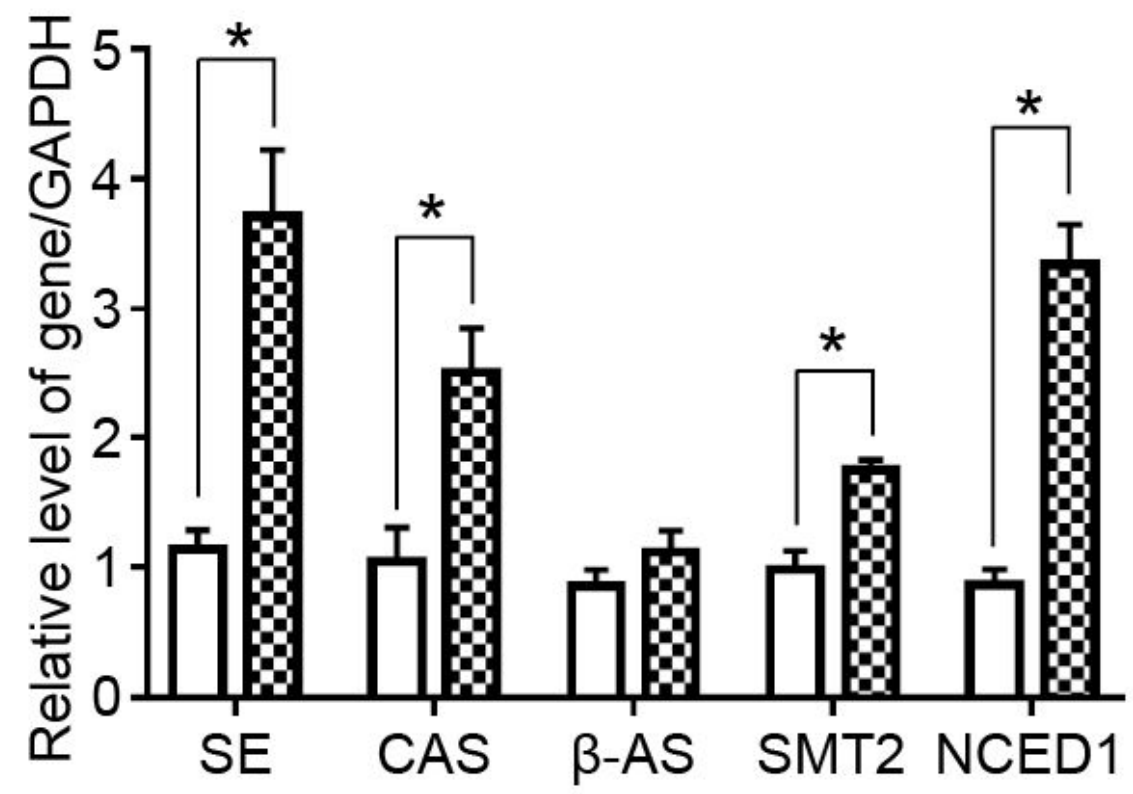

$\square$ before dehiscence $\mathbf{E}$ after dehiscence

Figure 5

The relative levels of expression of genes involved in the phytosterol and ginsenoside biosynthetic pathways. Asterisks indicate that the alteration in the gene is statistically significant after dehiscing (P-value $<0.005)$.

\section{Supplementary Files}

This is a list of supplementary files associated with this preprint. Click to download.

- Additionalfile.docx 